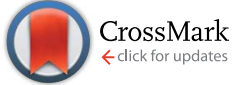

Cite this: RSC Adv., 2014, 4, 36377

Received 1st April 2014

Accepted 31st July 2014

DOI: $10.1039 / c 4 r a 02873 b$

www.rsc.org/advances

\section{Finely tuning oxygen functional groups of graphene materials and optimizing oxygen levels for capacitors $\uparrow$}

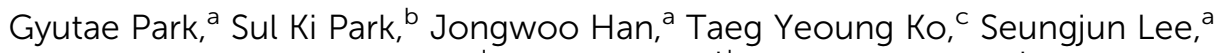 \\ Junghoon Oh, ${ }^{a}$ Sunmin Ryu, ${ }^{\text {cd }}$ Ho Seok Park ${ }^{\star b}$ and Sungjin Park ${ }^{\star a e}$
}

Oxygen-containing, chemically modified graphene (CMG) systems have been intensively investigated for various applications. The development of methods that allow fine control of the oxygen functionality would help better understand the mechanisms associated with CMGs, facilitate optimization of the material properties, and provide standards for chemical characterization purposes. Here, the authors report a new method for finely controlling the levels of oxygen in CMG materials based on the refluxing of aqueous colloidal suspensions of graphene oxide for specific reflux times, which does not require additional reducing agents. Chemical analysis confirmed that the oxygen levels can be finely controlled and they can provide spectroscopic tools to monitor the oxygen levels of CMG-based systems. This system was applied to help provide a fundamental foundation for the correlation between the oxygen groups and capacitive features.

\section{Introduction}

Chemically modified graphene (CMG) nano-platelet technology is attracting considerable interest in several application areas such as energy storage materials, ${ }^{1-4}$ electronic devices, ${ }^{5}$ chemical sensors, ${ }^{6}$ polymer composites, ${ }^{7}$ and electro-chemical catalysts. ${ }^{8,9}$ Oxygen-containing CMG systems have been intensively investigated for such applications because of their suitability for mass production, solution processability, and tolerances of further chemical modification. ${ }^{10,11}$ However, the chemical and physical properties of CMG materials critically depend on the amounts and chemical identities of oxygen functional groups. ${ }^{\mathbf{1 , 5 , 6 , 8 , 9 , 1 2 - 1 4}}$ Since it is extremely difficult to completely remove such groups, most CMGs contain some oxygen groups; $;^{1,8,13,15-28}$ consequently, new methods are required to finely control the oxygen functionalities. Elucidation of the mechanisms associated with CMGs, the optimization of

${ }^{a}$ Department of Chemistry and Chemical Engineering, Inha University, Incheon 402-751, Republic of Korea. E-mail: sungjinpark@inha.ac.kr; Fax: +82-32-867-5604; Tel: $+82-32-860-7677$

${ }^{b}$ School of Chemical Engineering, Sungkyunkwan University (SKKU), Suwon 440-746, Republic of Korea. E-mail: milleniumphs@gmail.com

'Department of Applied Chemistry, Kyung Hee University, Yongin, Gyeonggi 446-701, Republic of Korea

${ }^{d}$ Department of Chemistry, Pohang University of Science and Technology (POSTECH), Pohang, Kyungbuk 790-784, Republic of Korea

${ }^{e}$ Department of Chemistry, Inha University, Incheon 402-751, Republic of Korea

$\uparrow$ Electronic supplementary information (ESI) available: XPS, BET, CV, and SEM data. See DOI: $10.1039 / \mathrm{c} 4 \mathrm{ra02873b}$ materials properties, and the provision of tools for chemical characterizations have proved challenging.

By taking full advantage of oxygen functional groups, CMGs have been intensively studied as electrodes in supercapacitors (SCs) and rechargeable batteries, ${ }^{1-4,13,14,29}$ and as catalysts for oxygen reduction reactions (ORRs). ${ }^{12,30}$ Pseudocapacitance that originates from oxygen groups of electrode materials in SCs, ${ }^{31}$ the interaction between oxygen groups of CMGs and Li ions in batteries, and the electrochemical roles of oxygen groups during ORRs could be systematically investigated if a method can be devised to control the levels and types of oxygen functionalities. Hetero-atom (i.e. N, B, P and S)-doped graphenes derived from CMG-based systems have been intensively studied for possible applications in various areas. ${ }^{1,3,4,20,32,33}$ However, their overall performances could be influenced by oxygen groups as well as by other hetero-atoms because doped systems nearly always contain significant amounts of oxygen groups. ${ }^{1,28}$ Accordingly, it is very difficult to decouple the individual roles of hetero-atoms with oxygen groups; hence, the system would be more complicated. From this standpoint, new methods are in strong demand for finely controlling the oxygen groups of CMGs that include negligible hetero-atom compositions. Herein, we present a simple and environment friendly method of preparing graphene-based nanoplatelets containing controlled amounts of oxygen atoms based on the reflux times of aqueous suspensions of graphene oxide (GO). Furthermore, the sample series were comprehensively characterized to provide unprecedented spectroscopic tools to monitor the oxygen levels of CMG-based systems and systematically applied to understand a fundamental foundation of the correlation between oxygen groups and capacitive features. 


\section{Experimental}

\section{Preparation of GO}

GO was prepared by the modified Hummers method as previously reported..$^{18}$ Natural graphite ( $1 \mathrm{~g}, \mathrm{SP}-1$, Bay Carbon, USA) was added to a $250 \mathrm{~mL}$ flask filled with $50 \mathrm{~mL}$ of concentrated $\mathrm{H}_{2} \mathrm{SO}_{4}$ (Daejung, Korea) and $3.5 \mathrm{~g}$ of $\mathrm{KMnO}_{4}$ (99.3\%, Daejung, Korea) was added slowly to the flask in an ice-bath. The mixture was then stirred for $2 \mathrm{~h}$ at $30{ }^{\circ} \mathrm{C}$. Excess water was added to the reaction mixture in an ice-bath, and then $\mathrm{H}_{2} \mathrm{O}_{2}(30 \%$, SigmaAldrich, USA) was added slowly at room temperature until no gas was produced. The resulting mixture was then stirred for 12 $\mathrm{h}$, and then left unstirred to precipitate the GO particles. The supernatant was decanted and a $\mathrm{HCl}$ solution (10 vol\%) was added, and after centrifugation (Supra 22k, Hanil, Korea), the supernatant was washed five times with distilled water. The final mixture was then filtered and the filtrate was dried under vacuum at room temperature for $12 \mathrm{~h}$ to afford a brown GO powder.

\section{Preparation of a set of Re-G-O samples}

A homogeneous aqueous colloidal suspension ( $3 \mathrm{mg}$ of GO per 1 $\mathrm{mL}$ of $\mathrm{H}_{2} \mathrm{O}$ ) of exfoliated $\mathrm{G}-\mathrm{O}$ was produced in a flask by sonication (BRANSONIC 8510E-DTH, Output $250 \mathrm{~W}, 44 \mathrm{kHz}$ ). The $\mathrm{pH}$ of the suspension was 1.74. The flask then was immersed in an oil bath, and the brown suspension was stirred for the designated times (6 h (Re-G-O-1), 12 h (Re-G-O-2), 1 day (Re-G-O3), 2 days (Re-G-O-4), 3 days (Re-G-O-5), 5 days (Re-G-O-6), 1 week (Re-G-O-7), or 2 weeks (Re-G-O-8)) under reflux. The resultant mixtures were filtered through a glass filter and the filtrates obtained were thoroughly washed with water (>ten times), and then dried under vacuum at room temperature for $12 \mathrm{~h}$.

\section{Electrochemical tests}

CV and GCD measurements were carried out using a three electrode system. Each sample was used as a working electrode, $\mathrm{Ag} / \mathrm{AgCl}$ as reference electrode, and Pt wire as a counter electrode. $1 \mathrm{M}$ of aqueous $\mathrm{H}_{2} \mathrm{SO}_{4}$ solution was used as the electrolyte. All potentials were referred to the $\mathrm{Ag} / \mathrm{AgCl}$ reference electrode. The working electrode was composed of $85 \mathrm{wt} \%$ active material and $15 \mathrm{wt} \% \mathrm{PVDF}$ as a binder. These two components were mixed using a few drops of NMP as a solvent, ground in an agate mortar until a homogenous paste was obtained, and then pasted on a conductive aluminum sheet substrate. The electrode was dried overnight in an oven at $80{ }^{\circ} \mathrm{C}$ and used as the working electrode. The electrochemical characteristics were evaluated using a CHI 760D Electrochemical Workstation (CH Instruments) at room temperature. Electrochemical impedance spectroscopy (EIS) was evaluated in the frequency range from $10^{6}$ to $10^{-2} \mathrm{~Hz}$ at an amplitude of $10 \mathrm{mV}$ using a Solartron 1260. For practical measurements of the cyclic performance, a symmetrical two electrode configuration was assembled following our previous work. ${ }^{34}$ A Whatman glass microfiber filter used as the separator was immersed in $1 \mathrm{M}$ of aqueous $\mathrm{H}_{2} \mathrm{SO}_{4}$ solution. The symmetric devices were configured sandwiching an aqueous-electrolyte-soaked separator between two electrodes and clamped for a good contact. The specific capacitance of the electrodes were calculated using the following equation: $C_{\mathrm{s}}=\int \operatorname{Id} V(\Delta V \times \mathrm{M} \times \nu)$, where $C_{\mathrm{s}}$ is the specific capacitance of electrode $\left(\mathrm{F} \mathrm{g}^{-1}\right), V$ is the voltage $(\mathrm{V}), I$ is the current (A), $\nu$ is the scan rate $\left(\mathrm{V} \mathrm{s}^{-1}\right)$, and $M$ is the mass of the electrode material $(\mathrm{g})$.

\section{Instruments and measurements}

Elemental analysis was performed using a FLASH EA1112 (Thermo Electron, Italia) in a helium atmosphere to determine the $\mathrm{C}, \mathrm{N}, \mathrm{O}$, and $\mathrm{H}$ contents. XPS of powder samples was performed on an Angle-Resolved X-ray Photoelectron Spectrometer (Theta probe AR-XPS, Thermo Electron Cooperation, UK) using a MXR1 Gun-400 $\mu \mathrm{m} 15 \mathrm{keV}$ spectrometer. The FT-IR spectra were obtained from $\mathrm{KBr}$ pellets containing r-G-O powder samples using a FT-IR Vacuum Spectrometer (Bruker VERTEX $80 \mathrm{~V}$, Bruker, Germany). The MAS SSNMR measurements of the powder samples were performed using 6000 scans on a 600 MHz Varian unity INOVA (USA) using a HX-MAS probe and a 4 $\mathrm{mm}$ diameter zirconia rotor. The Raman and PL spectra were obtained under ambient conditions using a purpose-built micro-Raman setup, as previously described..$^{35}$ Briefly, an $\mathrm{Ar}$ ion laser beam $(514 \mathrm{~nm}, 0.033 \mathrm{~mW})$ was focused onto a spot of diameter $\sim 500 \mathrm{~nm}$ using an objective lens $(40 \times, \mathrm{NA}=0.60)$, and scattered light was then collected. The spectral resolution of the set up (defined as the line width of Rayleigh peaks) was 6 $\mathrm{cm}^{-1}$. Five spots were probed were probed per sample. The suspension $\mathrm{pH}$ values were measured using a $\mathrm{pH}$ meter (HI 8424, HANNA, USA).

\section{Results and discussion}

Oxygen functional groups in graphite oxide (GO) are generally introduced by the oxidation of graphite. ${ }^{36-38}$ Graphene oxide (GO) is produced by sonication of GO in water, and it has a wide range of oxygen functional groups, such as epoxy and hydroxyl groups on its basal planes and carboxyl and ketone groups at its edges. $^{39}$ The reduction of G-O using reductants and/or by applying external energy, such as heat, microwave, or light energy, is frequently used to reduce oxygen group levels and to produce reduced graphene oxide (rG-O). ${ }^{\mathbf{1 , 1 7 , 1 9 , 2 2 , 2 5 , 2 7 , 2 8 , 4 0 - 4 2}}$ However, most of these methods cannot finely control the oxygen levels. . $17,19,22,25,27,28,40$

Homogeneous aqueous colloidal suspensions of G-O were prepared by sonicating GO particles, and the $\mathrm{pH}$ of the suspensions prepared was around 1.7. This acidic property might be attributed to residual acids trapped in the interlayer galleries of GO particles and to the acidic protons of carboxyl acids on G-O. As shown in Fig. 1, we refluxed aqueous suspensions of G-O for different times ( 6 and $12 \mathrm{~h}$, and 1, 2, 3, 5, 7, and 14 days). After reflux for 1 day, G-O nano-platelets were well dispersed in the suspension (Fig. 1). Subsequently, black particles were generated, which is a common phenomenon when G-O is reduced. 


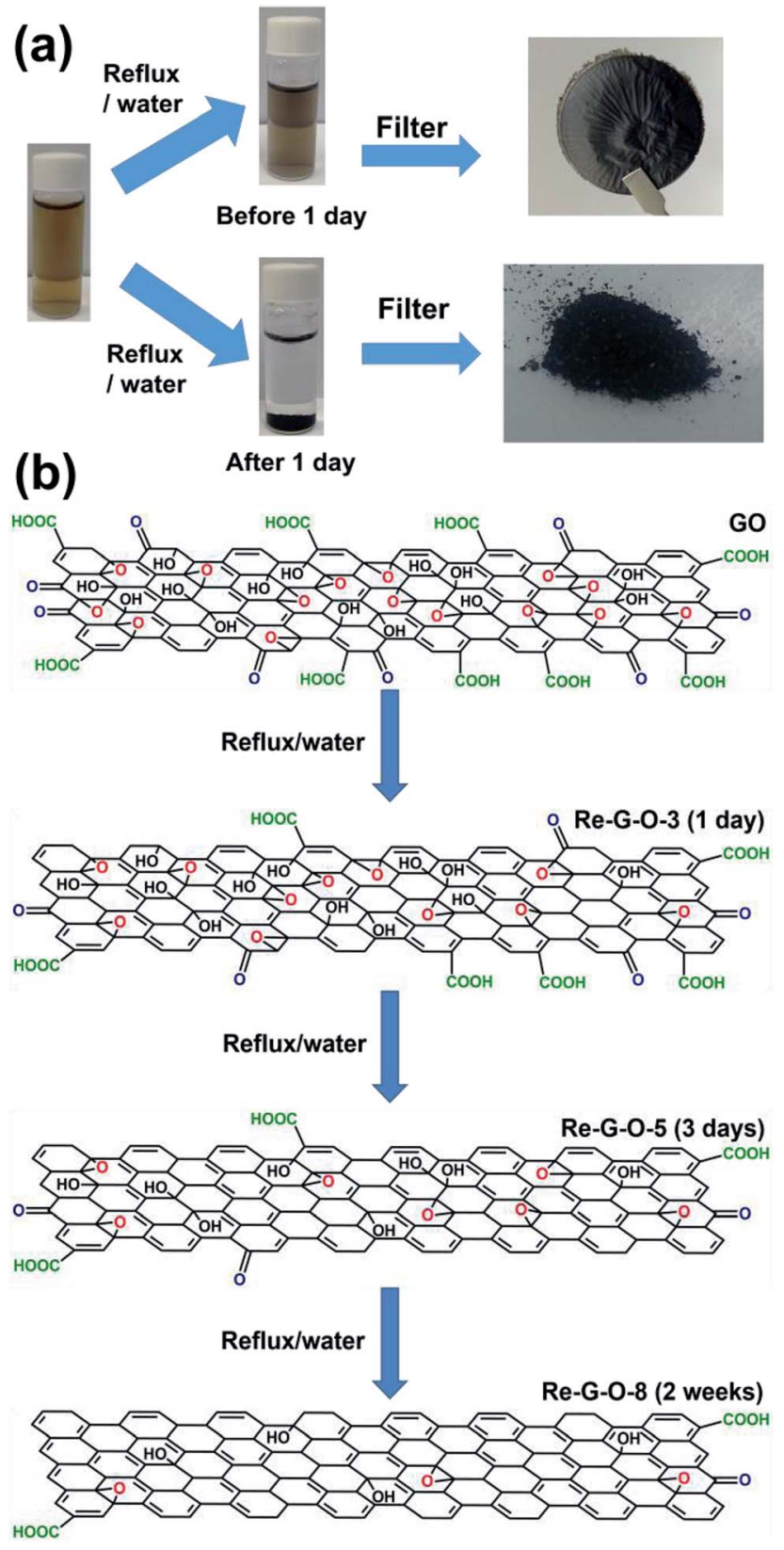

Fig. 1 Schematic diagram of the production of Re-G-O samples by refluxing aqueous suspensions of graphene oxide.

The C/O atomic ratio is typically used to determine the concentration of oxygen groups in graphene-based nano-platelets. ${ }^{\mathbf{1 , 1 9 , 2 2 , 2 7 , 2 8 , 4 0}}$ Interestingly, the $\mathrm{C} / \mathrm{O}$ ratio of refluxed $\mathrm{G}-\mathrm{O}$ (henceforth, Re-G-O) samples, which was measured by combustion-based elemental analysis, gradually increased in small increments as the reaction time increased, as shown in Fig. 2a. The $\mathrm{C} / \mathrm{O}$ ratio reached a steady state at around 7 after reflux for 2 weeks (the $\mathrm{C} / \mathrm{O}$ ratio range was between 1 and 7 ). The highest $\mathrm{C} / \mathrm{O}$ ratio (around 7 ) was comparable to that of $\mathrm{rG}-\mathrm{O}$ based materials produced using other methods. ${ }^{\mathbf{8 , 1 8 , 1 9 , 2 2 , 2 8 , 4 0 , 4 1} \mathrm{C} /}$ $\mathrm{O}$ ratios calculated by X-ray photoelectron spectroscopy (XPS) agreed well with the elemental analysis results (see (ESI $\dagger$ ) for
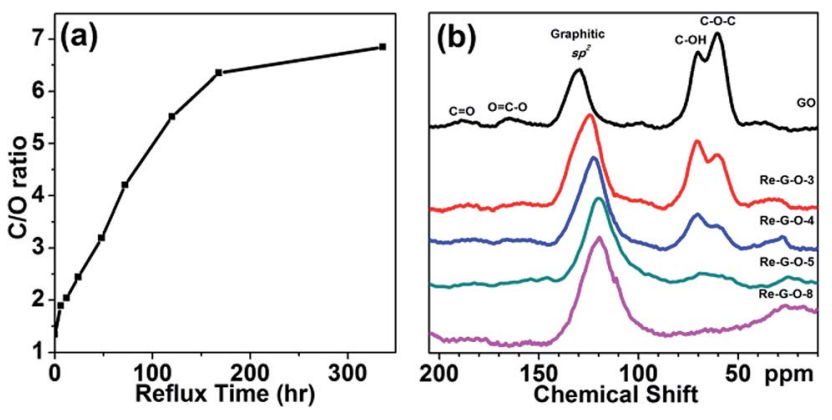

Fig. 2 Oxygen levels and NMR measurement of Re-G-O samples, (a) $\mathrm{C} / \mathrm{O}$ atomic ratio of $\mathrm{Re}-\mathrm{G}-\mathrm{O}$ samples measured by combustion-based elemental analysis with respect to reflux time, (b) NMR spectra of GO and selected Re-G-O samples.

Fig. S1 and Table S1). Importantly, this is the first example of a method that can produce graphene-based nano-platelets with finely controlled oxygen group levels using aqueous solution process. No hetero-atoms other than $\mathrm{O}$ atoms were found by elemental analysis and XPS. This result allows chemical and physical analysis of this system to show the effects of only oxygen groups on graphene-based networks without a disturbance from other hetero-atoms. In addition, this system produced rG-O by the reflux of G-O in water without the addition of reducing agents, indicating that it is a highly environmentally friendly method. These findings highlight the importance of a synthetic method for the mass production of rG-O materials containing a certain level of oxygen atoms.

We studied the chemical structures of Re-G-O samples using nuclear magnetic resonance (NMR) spectroscopy, Fourier transform infrared (FT-IR) spectroscopy, and Raman spectroscopy and XPS. NMR spectroscopy is one of the most powerful tools for elucidating the chemical structures of small molecules, and recently, solid state NMR (SSNMR) has shown great promise in the CMG-based material field. ${ }^{20,21,43,44}$ Fig. $2 \mathrm{~b}$ shows the magic angle spinning (MAS) SSNMR spectra of GO, Re-G-O3, Re-G-O-4, Re-G-O-5, and Re-G-O-8. The sampling labeling scheme was as follows: $6 \mathrm{~h}$ (Re-G-O-1), $12 \mathrm{~h}$ (Re-G-O-2), 1 day (Re-G-O-3), 2 days (Re-G-O-4), 3 days (Re-G-O-5), 5 days (Re-G-O6), 1 week (Re-G-O-7), and 2 weeks (Re-G-O-8). As previously assigned, ${ }^{\mathbf{4 3 4 4}}$ the spectrum of GO showed peaks for epoxy, hydroxyl, carboxyl, ketone, and $\mathrm{sp}^{2}$ carbon at 60.3, 70.2, 166.6, 189.0, and $129.4 \mathrm{ppm}$, respectively. As the reflux time increased, the intensities of the peaks corresponding to oxygen functional groups decreased, showing that G-O was reduced. Interestingly, a peak of GO at $129.3 \mathrm{ppm}$, corresponding to $\mathrm{sp}^{2}$ carbons was gradually shifted to $124.6 \mathrm{ppm}$ for Re-G-O-3, $122.6 \mathrm{ppm}$ for ReG-O-4, 120.0 ppm for Re-G-O-5, and 119.7 ppm for Re-G-O-8, respectively. This was attributed to elongated conjugation generated by the restoration of $\mathrm{sp}^{2}$ carbon networks from $\mathrm{sp}^{3}$ carbons during reflux. ${ }^{20,43}$ As the peak positions were shifted, their $\mathrm{C} / \mathrm{O}$ ratios increased. The observation of this peak position corresponding to $\mathrm{sp}^{2}$ carbons could provide the relation between such NMR peaks and the oxygen levels (see Fig. S2 $\dagger$ ). Epoxy and hydroxyl groups were significantly reduced after 2 weeks of reflux. Although the intensities of the peaks 
corresponding to ketone and carboxyl decreased slightly, it was not possible to quantify results due to noise. Nevertheless, we were able to deduce that epoxy groups were reduced faster than hydroxyl groups. While $\mathrm{C}-\mathrm{O}$ moieties were reduced, $\mathrm{C}=\mathrm{O}$ moieties, such as carboxyl and ketone groups, were only slightly reduced. It is difficult to observe this type of removal sequence using other chemical analysis tools, such as XPS or FT-IR due to substantial overlapping of the peaks., ${ }^{\mathbf{1 , 8 , 1 8 , 2 2 , 4 1 , 4 5}}$

As shown in Fig. $\mathrm{S} 3, \uparrow$ the components for $\mathrm{C}-\mathrm{O}$ moieties at around $285 \mathrm{eV}$ and for $\mathrm{C}=\mathrm{O} / \mathrm{C}(\mathrm{O}) \mathrm{O}$ moieties at 288-289 eV were observed in the XPS C1s spectrum of GO., ${ }^{1,18,23}$ The series of C1s spectra for all Re-G-O samples and for GO demonstrated the gradual removal of these oxygen moieties as reflux progressed. Deconvolution of C1s spectra of selected samples (GO, Re-G-O4, Re-G-O-5, Re-G-O-6, and Re-G-O-8) produced results consistent with NMR with respect to the reduction by reflux (Fig. S3 $\dagger$ ). A component corresponding to the $\mathrm{C}-\mathrm{O}$ moieties was first removed and was significantly removed after 2 weeks of reflux. Slight reduction in the $\mathrm{C}=\mathrm{O} / \mathrm{C}(\mathrm{O}) \mathrm{O}$ moieties was observed by XPS.

Fig. 3a shows the FT-IR spectra of the series of Re-G-O samples and GO. As reflux time increased, a peak at $\sim 3400$ $\mathrm{cm}^{-1}$ corresponding to the $\mathrm{O}-\mathrm{H}$ stretching band, ${ }^{\mathbf{4 6}}$ gradually decreased, suggesting the removal of hydroxyl groups from the basal planes of G-O. Components of the $\mathrm{C}-\mathrm{O}$ stretching bands at $1,417,1,224$, and $1053 \mathrm{~cm}^{-1}$, which were attributed to hydroxyl and epoxy groups, also decreased during reflux. ${ }^{16,45,46}$ After 3 days of reflux, the intensities of these peaks decreased significantly, which concurs with our NMR results. Peaks corresponding to $\mathrm{C}=\mathrm{C}$ stretching provide particularly interesting information. ${ }^{46}$ The peak at $1622 \mathrm{~cm}^{-1}$ in the spectrum of GO
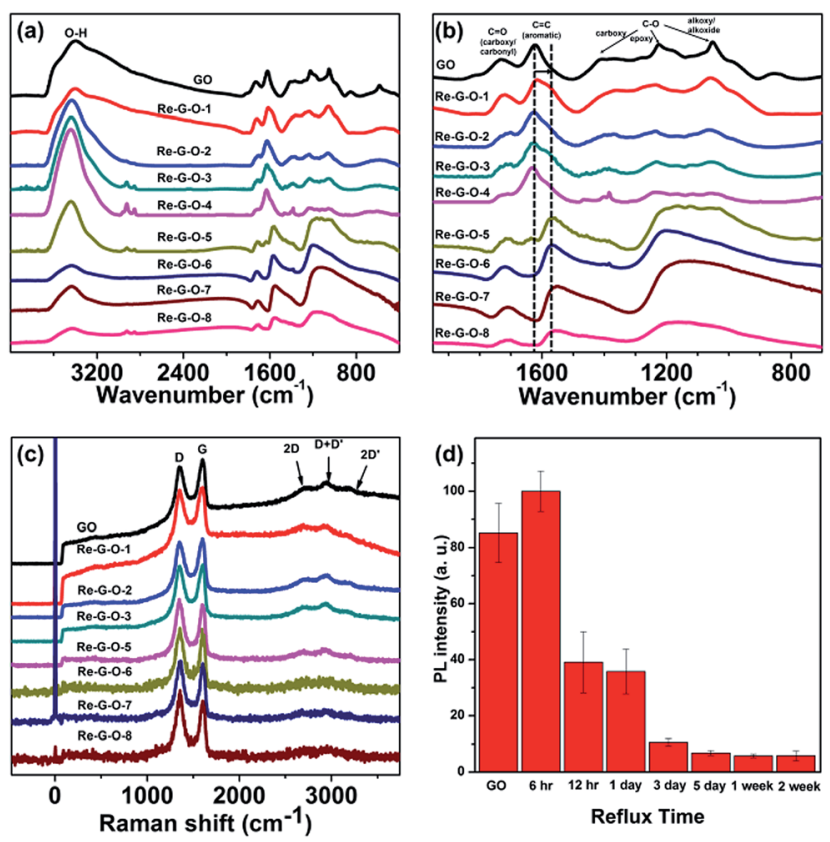

Fig. 3 Spectroscopic data of GO and a series of Re-G-O samples, (a) FT-IR spectra at all region, (b) FT-IR spectra of the selected region, (c) Raman spectra, (d) PL measurements. and in those of early stage-reduction samples (Re-G-O-1-4) was shifted to $1569 \mathrm{~cm}^{-1}$ in the spectra of the late stage-reduction samples (Re-G-O-5-8), as shown in Fig. 3b (note that the Re-GO-5 sample was refluxed for 3 days). It is generally accepted that $\mathrm{sp}^{2}$ carbon networks are restored during the reduction of the oxidized carbon network of G-O. Peak shifting in the FT-IR spectra suggests that the peak position corresponding to the $\mathrm{C}=\mathrm{C}$ stretch of $\mathrm{sp}^{2}$ networks, bearing oxygen groups with long range ordering, represents the degree of reduction. The $\mathrm{C} / \mathrm{O}$ ratio of Re-G-O-5 ( 4.3 by elemental analysis and 4.2 by XPS) could be a standard to determine the peak shift. A peak at 1731 $\mathrm{cm}^{-1}$, corresponding to the $\mathrm{C}=\mathrm{O}$ stretching band, was slightly reduced after 2 weeks of reflux. Note that no peak was observed at $\sim 1150 \mathrm{~cm}^{-1}$, corresponding to the $\mathrm{C}-\mathrm{H}$ in-plane bending of phenyl groups, ${ }^{46}$ in the spectrum of GO. However, a broad peak at the region increased after 3 days of reflux (Re-G-O-5-8), suggesting that the number of edge-state $\mathrm{sp}^{2}$ carbons, including the outer regions of nano-platelets and defect sites in the inner planes, increased during reflux.

Raman and photoluminescence (PL) spectroscopy also supported the structural changes caused by the stepwise reduction of G-O. The spectra in Fig. 3c show D and G peaks characteristic of defective $\mathrm{sp}^{2}$-rich carbon materials. ${ }^{47,48}$ The $\mathrm{G}$ peak originates from in-plane $\mathrm{C}-\mathrm{C}$ stretching, whereas the $\mathrm{D}$ peak is due to double resonance scattering activated by structural defects. However, because of disorder-induced spectral broadening, another disorder-related peak $\left(\mathrm{D}^{\prime}\right.$ at $\left.1620 \mathrm{~cm}^{-1}\right)$ could not be resolved from the $\mathrm{G}$ peak. In addition to a $\mathrm{D}$ and $\mathrm{D}^{\prime}$ combination peak (denoted $\mathrm{D}+\mathrm{D}^{\prime}$ ), a Raman spectrum of G-O exhibited broad $2 \mathrm{D}$ and $2 \mathrm{D}^{\prime}$ peaks (overtones of $\mathrm{D}$ and $\mathrm{D}^{\prime}$ modes). As G-O was reduced, the $\mathrm{D}$-to-G peak area ratio $\left(I_{\mathrm{D}} / I_{\mathrm{G}}\right)$ gradually increased (Fig. S4†). This finding apparently contradicts the notion that reduction removes defects and leads to recovery of the $\mathrm{sp}^{2}$ carbons state. ${ }^{49}$ Since the $\mathrm{D}$ peak requires the presence of defects, $I_{\mathrm{D}} / I_{\mathrm{G}}$ increases with increasing defect density in graphene, ${ }^{50}$ and when the density exceeds a certain threshold and the amorphous phase becomes dominant, the ratio starts to decrease due to the severe loss of graphene-like order. ${ }^{\mathbf{4 8 , 5 0}}$ Thus, the observed rise in $I_{\mathrm{D}} / I_{\mathrm{G}}$ indicates that all samples belong to the latter case and that reflux indeed recovered $\mathrm{sp}^{2}$ carbon. Decreases in the line widths of the $\mathrm{G}$ and $\mathrm{D}$ peaks corroborate this conclusion (Fig. $\mathrm{S} 5 \dagger$ ), since their large line widths are due to a wide distribution of structural orders caused by defects. ${ }^{47}$ The increase in G frequency upon reduction is consistent with previous studies on defective graphite (Fig. S6 $\dagger$ ). ${ }^{47}$ Last but not the least, the important feature is that reduction greatly attenuated the PL signal (a broad background in Fig. 3c), which makes the Re-G-O samples less luminescent. In fact, the PL intensity determined in the $1700-2300 \mathrm{~cm}^{-1}(564-584 \mathrm{~nm})$ region increased initially and then decreased during reflux (Fig. 3d), which is fully consistent with a previous observation by Eda et $a l .{ }^{49}$ In this previous study, the early stage of reduction was found to increase the density of the isolated nanocrystalline domains responsible for the observed PL and further reduction recovered the $\mathrm{sp}^{2}$ network sufficiently to quench PL. Our studies have shown that Raman and PL spectroscopy can serve as a convenient and reliable means of 
assessing the degree of chemical reaction in highly crystalline graphene. ${ }^{51,52}$

As described above, all chemical analysis results supported the successful reduction of G-O by reflux. A huge amount of effort has been expended on the development of a simple, environmentally friendly solution process to reduce G-O. However, efficiency was only obtained at the expense of using toxic and/or hazardous chemicals..$^{1,22,27,28}$ Thus, the removal of such chemicals is a critical issue for further applications. Consequently, it is noteworthy that the present study describes the generation of rG-O by reflux of G-O in water without the addition of any chemicals and without a subsequent purification step to remove the reducing agents.

Acidic protons can reduce the oxygen functionalities of small molecules via dehydration. In a previous report, ${ }^{24}$ hydrothermal treatment of G-O under weak acidic conditions $(\mathrm{pH} \sim 4)$ slightly reduced the oxygen levels, which were much higher than those in our system. Consequently, the acidic condition ( $\mathrm{pH}$ of 1.7) of G-O suspensions in our system is largely responsible for the reduction by reflux. Refluxing under different acidic conditions also showed similar reduction behaviour (Table S2 $\dagger$ ). It would be interesting to observe reducing behaviors under basic conditions to better understand the role of $\mathrm{pH}$. However, it is known that strong bases, such as $\mathrm{KOH}$, and weak bases, such as amine derivatives, also reduce G-O, ${ }^{18,21,53}$ which would obviously prevent an investigation of the role played by $\mathrm{pH}$ alone.

Electron-rich nucleophiles, such as hydrazine and its derivatives, ${ }^{28}$ amine derivatives, ${ }^{53} \mathrm{HI},{ }^{54} \mathrm{NaBH}_{4},{ }^{22}$ and $\mathrm{KOH},{ }^{18,21}$ are capable of reducing G-O. It has been suggested that reduction could be progressed by nucleophilic attack on the electron deficient $\alpha$-carbons of oxygen groups. It was recently reported that electron-deficient electrophiles, such as $\mathrm{BH}_{3}$-based adducts can reduce G-O. ${ }^{1}$ In this case, electrophiles are attacked by the lone pair electrons of G-O oxygen atoms. In our system, it would be an electrophilic reduction by acidic protons and electrophiles. Huge amounts of water and acids are typically used during the production of GO, and it is well known that a number of water and acid molecules are trapped in the interlayer galleries by the strong interaction with the oxygen groups of GO. ${ }^{18,55,56}$ Accordingly, these trapped acidic moieties could be a source of acidic protons.

To demonstrate the superiority of a series of graphene nanoplatelets containing finely controlled amounts oxygen groups, we tested the SC performance of these electrode materials. The pristine and CMG-based materials serve as a potential electrode for the use in SCs due to their unique physical and chemical properties. Since the SCs store charges at the electrochemical double layer (EDL) through a non-faradic electrostatic interaction between electrons and ions ${ }^{57}$ the capacitance of graphenes are promising due to their large surface area and electronic conductivity. The oxygen-containing groups of rG-O can generate surface redox capacitance, so-called pseudocapacitance, through a faradic process for enhanced capacitance. ${ }^{58}$ Moreover, oxygen functional groups can offer good dispersion capability and processability for an electrode assembly and wettability of ions on the adsorption sites can increase due to the hydrophilic nature of polar oxygen-containing groups. ${ }^{59}$
Notwithstanding these advantages of surface chemistry, the intrinsic conjugated $\mathrm{sp}^{2}$ structure of graphene could be disrupted, indicating that the deterioration of electrical conductivity originated from the high density of defects. Alternatively, severe reduction of G-O for the restoration of conjugation is required to remove the oxygen defect sites, yet it can be restacked and aggregated. ${ }^{11}$ Consequently, our Re-G-O materials will be highly useful to determine optimum conditions of rG-O materials in SCs.

The electrochemical behavior and capacitor performance of Re-G-O samples were evaluated by cyclic voltammetry (CV) and galvanostatic charge/discharge tests (GCD) in a potential window from -0.2 to $1.0 \mathrm{~V}$ versus $\mathrm{Ag} / \mathrm{AgCl}$ in a $1 \mathrm{M}$ aqueous $\mathrm{H}_{2} \mathrm{SO}_{4}$ solution as an electrolyte. The current densities of GO and Re-G-O-1-4 samples were too small to be measurable for the capacitive behavior. As shown in the irregular CV shape with high overpotential and without a redox peak on CV (see Fig. S7 $\dagger$ ), they did not behave as capacitors but as resistors due to a disruption of the intrinsic conjugated $\mathrm{sp}^{2}$ structure. This indicates that despite numerous oxygen-containing groups that can act as electroactive sites, the deep oxidation of GO and Re-G0-1-4 samples prevents the occurrence of charge separation (or discharging) and accumulation (or charging) during a redox charge transfer due to a deterioration of the electrical properties.

As shown in the CV curves of Fig. 4a, all Re-G-O-5-8 samples exhibited similar rectangular shapes at a rate of $10 \mathrm{mV} \mathrm{s}^{-1}$, when the degree of reduction is beyond a critical value (C/O ratio of Re-G-O-5: 4.2), indicating that a meaningful amount of capacitive current is stored in a faradic manner. One pair of redox waves at 0.32 and $0.44 \mathrm{~V}$ and asymmetric CV shape at the end of potential window originated from the electroactivity of oxygen functional groups such as epoxy, carboxy carbonyl and alkoxy alkoxide (see SI).

The specific capacitances of the Re-G-O-5-8 samples obtained from GCD curves were calculated to be 113.8, 140.5, 135.0, and $97.3 \mathrm{~F} \mathrm{~g}^{-1}$ at a specific current of $1 \mathrm{~A} \mathrm{~g}^{-1}$, respectively (Fig. 4b). The specific capacitances of the Re-G-O samples obtained from the GCD curves showed the same trend as those of the CV curves, but each value was slightly different because of the respective measurement conditions. In particular, Re-G-O-6 showed the highest capacitance at all scan rates (Fig. S8 $\dagger$ ) and the highest rate retention among the Re-G-O samples. Moreover, Re-G-O samples revealed a slightly distorted, nonlinear shape in the discharge region at a voltage range of $0.4 \mathrm{~V}$, which is indicative of the typical pseudocapacitive properties of the respective oxygen functional groups. In order to further investigate the capacitive features arising from oxygen functional groups, the rate capability of Re-G-O samples were measured (Fig. 4c). The rate capability was estimated by increasing the scan rates from 10 to $200 \mathrm{mV} \mathrm{s}^{-1}$ by a factor of 20 . The capacitance retentions of Re-G-O-5-8 and hrG-O were $68 \%, 80 \%$, $65 \%, 70 \%$, and $39 \%$, respectively (Fig. S9†). For the case of the cyclic performance (measured by GCD curves in a two-electrode symmetric configuration at $5 \mathrm{~A} \mathrm{~g} \mathrm{~g}^{-1}$ ), Re-G-O-6 retained $95 \%$ of initial capacitances after 1000 cycles, indicating good electrochemical stability, which is comparable to $96 \%$ and $99 \%$ 

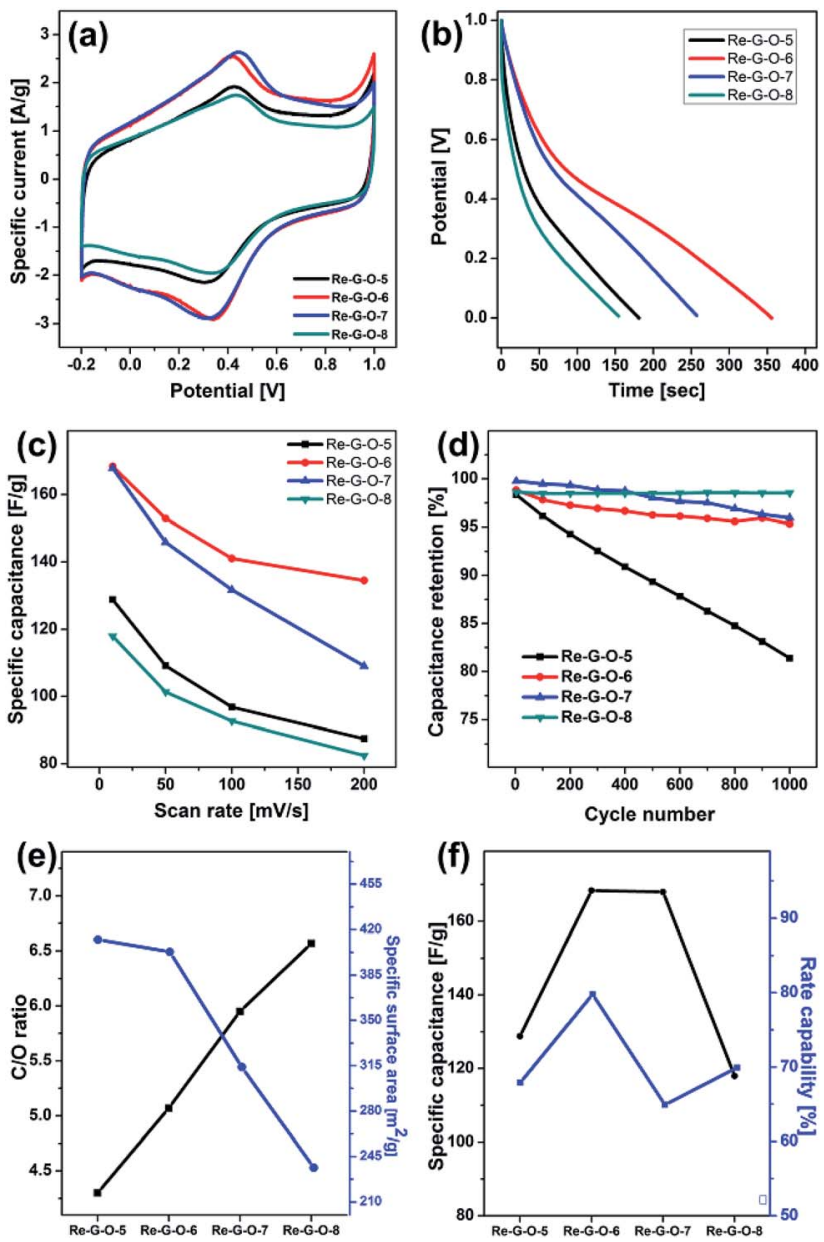

Fig. 4 (a) CV curves of Re-G-O-5-8 at a scan rate of $10 \mathrm{mV} \mathrm{s}^{-1}$ (b) GCD curves of Re-G-O-5-8 at a specific current of $1 \mathrm{~A} \mathrm{~g}^{-1}$. (c) Rate capabilities of Re-G-O-5-8 measured at specific currents from 10 to $200 \mathrm{mV} \mathrm{g}^{-1}$. (d) Cyclic stabilities of Re-G-0-5-8 measured at a specific current of $5 \mathrm{~A} \mathrm{~g}^{-1}$. Variation of the materials properties of a series of $\mathrm{Re}-\mathrm{G}-\mathrm{O}$ samples; (e) $\mathrm{C} / \mathrm{O}$ ratio and surface area and (f) specific capacitance and rate capability.

observed for Re-G-O-7 and Re-G-O-8, respectively (Fig. 4d). Consequently, the GCD curves of Re-G-O-6-8 were not significantly distorted after 1000 cycles of charge/discharge (Fig. S10†).

Despite a higher $\mathrm{C} / \mathrm{O}$ ratio and faster electronic transport, the rate capabilities of Re-G-O-7 and Re-G-O-8 were lower compared to that of Re-G-O-6 due to the slower ion diffusion originated from the restacking-induced pore blocking at a severe reduction degree and the less accessible surface area at high rates. On the other hand, the removal of oxygen functional groups that can act as electrochemically degradable defects is beneficial for improving the cyclic stability, which highlights the significance of delicately controlled oxygen functional groups for the optimum capacitive performance.

The effect of oxygen functional groups on the surface textures and chemistry have to be investigated to understand how the capacitive performances can be controlled by the suggested chemistry. As the reflux time increased, C/O ratio was increased and then, saturated to $\sim 7$. It is worth noting that restacking of nano-platelets during a reduction process results in a loss of available surface area and ion diffusion pathway due to the blocking of mesopores arising from intersheet voids. EIS analysis is a powerful tool for understanding the electrochemical behaviors of SC at the bulk and interface. As shown in the Nyquist plots of Re-G-O-5-8 in the frequency range of $10^{6}$ to $10^{-2} \mathrm{~Hz}$ at an amplitude of $10 \mathrm{mV}$ (Fig. S11†), Re-G-O-7 and ReG-O-8 samples have lower equivalent series resistance (ESR) values than Re-G-O-5 and Re-G-O-6 samples, which are derived from the intersection point on the real axis at high frequency (Table S3†). This indicates that the Re-G-O-7 and Re-G-O-8 samples retain better electrical conductivity associated with a higher C/O ratio than the Re-G-O-5 and Re-G-O-6 samples. The semicircular behavior in the high- to mid-frequency region corresponds to the interfacial charge transfer resistance $\left(R_{\mathrm{ct}}\right)$ originating from the charge-transfer process occurring at the electrode-electrolyte interface. The $R_{\mathrm{ct}}$ values increased with respect to the reduction time, indicating that the abundant oxygen functional groups lead to a favourable interfacial interaction during pseudocapacitive charging/discharging. ${ }^{34}$ Moreover, Re-G-O-5 and Re-G-O-6 samples have a higher surface area and more crumpled morphology relative to the Re-G-O-7 and Re-G-O-8 samples (see Table $\mathrm{S} 4 \uparrow$ and scanning electron microscopy images in Fig. S12†). Therefore, the capacitive performances were optimized at Re-G-O-6 with the moderate C/ $\mathrm{O}$ ratio and large surface area due to the synergistic coupling of the pseudocapacitance of oxygen functional groups with the large accessible area by the crumpled morphology.

The capacitances of all Re-G-O-5-8 samples retained $65-80 \%$ of the initial values in the same range in spite of lower $\mathrm{C} / \mathrm{O}$ ratio compared to that of hrG-O. In particular, Re-G-O-6 with C/O ratio of 5.5 and a surface area of $403 \mathrm{~m}^{2} \mathrm{~g}^{-1}$ showed the highest capacitance among the Re-G-O samples. Compared with the negligible capacitance of Re-G-O-1-4, a C/O ratio above the critical value of Re-G-O-5 could be crucial for revealing the pseudocapacitance, resulting from the sufficient electronic transport during a charge accumulation and separation process. At a moderate amount of oxygen containing groups and sufficient $\mathrm{C} / \mathrm{O}$ ratio controlled by reflux reduction chemistry, the optimum capacitance values of Re-G-O-6 were associated with the synergistic coupling of oxygen functional groups with available surface area and degree of reduction (Fig. 4e and f). Furthermore, substantial modification of Re-G-O samples to incorporate oxygen containing groups increases the wettability of the electrode for the improved overall performance of the capacitor. These results highlight the importance of refluxbased reduction chemistry on the tuning of oxygen containing groups while minimizing the restacking of nano-platelets.

\section{Conclusions}

We described a new method to finely control the levels of oxygen atoms in CMG nano-platelets based on the reflux of aqueous colloidal suspensions of G-O under acidic conditions for different times. Using this method, oxygen groups were removed from G-O without the use of reductants, and rG-O 
materials (Re-G-O) were generated. The C/O ratios of the Re-G-O series were found to increase gradually from $\sim 1$ to 7 with increasing reflux time, and the $\mathrm{C} / \mathrm{O}$ ratios reached a steady state of $\sim 7$ after 2 weeks of reflux.

The chemical identities of the CMG materials at each stage were intensively characterized by NMR, FT-IR, Raman, and PL spectroscopy and XPS. The results confirmed that oxygen amounts can be finely controlled, and they were consistent with respect to the chemical identities. This study provides a means of understanding the relationships between spectroscopic data and the chemical identities of oxygen atoms in CMG-based systems. The pseudocapacitive features of the Re-G-O prepared by reflux chemistry were determined by the $\mathrm{C} / \mathrm{O}$ ratio and specific surface area. The optimized capacitive performances of Re-G-O samples were found at a specific reflux time due to the synergistic coupling of oxygen functional groups with the $\mathrm{C} / \mathrm{O}$ ratio and surface area. The present study provides a straightforward means of producing tailor-made CMG materials containing certain oxygen components. We believe the technique will aid in our understanding of CMG materials and allow optimization of the materials properties for specific applications, particularly, for energy storage and conversion systems, electro- and photo-chemical catalysis, electronic devices, chemical/bio sensors, and polymer composites.

\section{Acknowledgements}

S.P. thanks the Busan Center, Korean Basic Science Institute (KBSI) for the XPS analysis. This work was supported by grants from INHA University, and grants from the Center for Advanced Soft Electronics via the Global Frontier Research Program of the Korean Ministry of Science, ICT and Future Planning (Grant no. 2013M3A6A5073173), and from the International Cooperation of the Korean Institute of Energy Technology Evaluation and Planning (KETEP) under the Korean Ministry of Knowledge Economy (Grant no. 20128510010050). H.P. acknowledges the support from the National Research Foundation (NRF) funded by the Korean Government (MEST) (20090063004) and NRF2010-C1AAA001-0029018. S.R. acknowledges the support from the National Research Foundation of Korea (NRF-20110010863).

\section{Notes and references}

1 J. Han, L. L. Zhang, S. Lee, J. Oh, K. Lee, J. R. Potts, J. Ji, X. Zhao, R. S. Ruoff and S. Park, ACS Nano, 2013, 7, 19-26.

2 U. N. Maiti, J. Lim, K. E. Lee, W. J. Lee and S. O. Kim, Adv. Mater., 2014, 26, 615-619.

3 U. N. Maiti, W. J. Lee, J. M. Lee, Y. Oh, J. Y. Kim, J. E. Kim, J. Shim, T. H. Han and S. O. Kim, Adv. Mater., 2014, 26, 40-67.

4 W. JunáLee, T. HoonáHwang, J. OkáHwang, H. WookáKim, H. YoungáJeong, T. HeeáHan, J. YoungáKim, J. WookáChoi and S. OukáKim, Energy Environ. Sci., 2014, 7, 621-626.

5 G. Eda, G. Fanchini and M. Chhowalla, Nat. Nanotechnol., 2008, 3, 270-274.
6 C. Lu, H. Yang, C. Zhu, X. Chen and G. Chen, Angew. Chem., 2009, 121, 4879-4881.

7 S. Stankovich, D. A. Dikin, G. H. Dommett, K. M. Kohlhaas, E. J. Zimney, E. A. Stach, R. D. Piner, S. T. Nguyen and R. S. Ruoff, Nature, 2006, 442, 282-286.

8 F. Liu and D. Xue, Chem.-Eur. J., 2013, 19, 10716-10722.

9 X. Yang, H. Cui, Y. Li, J. Qin, R. Zhang and H. Tang, ACS Catal., 2013, 3, 363-369.

10 D. R. Dreyer, S. Park, C. W. Bielawski and R. S. Ruoff, Chem. Soc. Rev., 2010, 39, 228-240.

11 S. Park and R. S. Ruoff, Nat. Nanotechnol., 2009, 4, 217-224. 12 Y. Liang, Y. Li, H. Wang, J. Zhou, J. Wang, T. Regier and H. Dai, Nat. Mater., 2011, 10, 780-786.

13 B. Zhao, P. Liu, Y. Jiang, D. Pan, H. Tao, J. Song, T. Fang and W. Xu, J. Power Sources, 2012, 198, 423-427.

14 H. Kim, H. Lim, S. Kim, J. Hong, D. Seo, D. Kim, S. Jeon, S. Park and K. Kang, Sci. Rep., 2013, 3, 1506.

15 D. C. Marcano, D. V. Kosynkin, J. M. Berlin, A. Sinitskii, Z. Sun, A. Slesarev, L. B. Alemany, W. Lu and J. M. Tour, ACS Nano, 2010, 4, 4806-4814.

16 C. Hontoria-Lucas, A. Lopez-Peinado, J. d. D. LópezGonzález, M. Rojas-Cervantes and R. Martin-Aranda, Carbon, 1995, 33, 1585-1592.

17 H. Guo, X. Wang, Q. Qian, F. Wang and X. Xia, ACS Nano, 2009, 3, 2653-2659.

18 S. Park, J. An, R. D. Piner, I. Jung, D. Yang, A. Velamakanni, S. T. Nguyen and R. S. Ruoff, Chem. Mater., 2008, 20, 65926594.

19 C. Nethravathi and M. Rajamathi, Carbon, 2008, 46, 19941998.

20 S. Park, Y. Hu, J. O. Hwang, E. Lee, L. B. Casabianca, W. Cai, J. R. Potts, H. Ha, S. Chen and J. Oh, Nat. Commun., 2012, 3, 638.

21 X. Fan, W. Peng, Y. Li, X. Li, S. Wang, G. Zhang and F. Zhang, Adv. Mater., 2008, 20, 4490-4493.

22 H. Shin, K. K. Kim, A. Benayad, S. Yoon, H. K. Park, I. Jung, M. H. Jin, H. Jeong, J. M. Kim and J. Choi, Adv. Funct. Mater., 2009, 19, 1987-1992.

23 S. Park, J. An, J. R. Potts, A. Velamakanni, S. Murali and R. S. Ruoff, Carbon, 2011, 49, 3019-3023.

24 S. Choudhary, H. P. Mungse and O. P. Khatri, Chem. - Asian J., 2013, 8, 2070.

25 A. V. Murugan, T. Muraliganth and A. Manthiram, Chem. Mater., 2009, 21, 5004-5006.

26 C. K. Chua and M. Pumera, Chem. - Eur. J., 2013, 19, 20052011.

27 J. Han, S. Lee, L. L. Zhang, J. Oh, J. R. Potts, R. D. Piner, R. S. Ruoff and S. Park, Solid State Sci., 2013, 25, 1-5.

28 S. Stankovich, D. A. Dikin, R. D. Piner, K. A. Kohlhaas, A. Kleinhammes, Y. Jia, Y. Wu, S. T. Nguyen and R. S. Ruoff, Carbon, 2007, 45, 1558-1565.

29 S. Yu, D. E. Conte, S. Baek, D. Lee, S. Park, K. J. Lee, Y. Piao, Y. Sung and N. Pinna, Adv. Funct. Mater., 2013, 23, 42934305.

30 Y. Li, Y. Zhao, H. Cheng, Y. Hu, G. Shi, L. Dai and L. Qu, J. Am. Chem. Soc., 2011, 134, 15-18.

31 D. S. Su and R. Schlögl, ChemSusChem, 2010, 3, 136-168. 
32 Z. Liu, F. Peng, H. Wang, H. Yu, W. Zheng and J. Yang, Angew. Chem., 2011, 123, 3315-3319.

33 Z. Yang, Z. Yao, G. Li, G. Fang, H. Nie, Z. Liu, X. Zhou, X. Chen and S. Huang, ACS Nano, 2011, 6, 205-211.

34 B. G. Choi, Y. S. Huh, W. H. Hong, H. J. Kim and H. S. Park, Nanoscale, 2012, 4, 5394-5400.

35 J. E. Lee, G. Ahn, J. Shim, Y. S. Lee and S. Ryu, Nat. Commun., 2012, 3, 1024.

36 B. C. Brodie, Philos. Trans. R. Soc. London, 1859, 149, 249259.

37 L. Staudenmaier, Ber. Dtsch. Chem. Ges., 1898, 31, 14811487.

38 W. S. Hummers,Jr and R. E. Offeman, J. Am. Chem. Soc., 1958, 80, 1339.

39 H. He, J. Klinowski, M. Forster and A. Lerf, Chem. Phys. Lett., 1998, 287, 53-56.

40 M. Choucair, P. Thordarson and J. A. Stride, Nat. Nanotechnol., 2008, 4, 30-33.

41 O. C. Compton, B. Jain, D. A. Dikin, A. Abouimrane, K. Amine and S. T. Nguyen, ACS Nano, 2011, 5, 4380-4391.

42 Y. Zhang, L. Guo, S. Wei, Y. He, H. Xia, Q. Chen, H. Sun and F. Xiao, Nano Today, 2010, 5, 15-20.

43 W. Gao, L. B. Alemany, L. Ci and P. M. Ajayan, Nat. Chem., 2009, 1, 403-408.

44 W. Cai, R. D. Piner, F. J. Stadermann, S. Park, M. A. Shaibat, Y. Ishii, D. Yang, A. Velamakanni, S. J. An and M. Stoller, Science, 2008, 321, 1815-1817.

45 S. Park, K. Lee, G. Bozoklu, W. Cai, S. T. Nguyen and R. S. Ruoff, ACS Nano, 2008, 2, 572-578.

46 J. B. Lambert, H. F. Shurvell, D. A. Lightner, R. G. Cooks, Organic Structural Spectroscopy, Prentice-Hall, Inc., USA, 1988.
47 A. Ferrari and J. Robertson, Phys. Rev. B: Condens. Matter Mater. Phys., 2000, 61, 14095.

48 S. Ryu, J. Maultzsch, M. Y. Han, P. Kim and L. E. Brus, ACS Nano, 2011, 5, 4123-4130.

49 G. Eda, Y. Lin, C. Mattevi, H. Yamaguchi, H. Chen, I. Chen, C. Chen and M. Chhowalla, Adv. Mater., 2010, 22, 505-509.

50 M. M. Lucchese, F. Stavale, E. Ferreira, C. Vilani, M. Moutinho, R. B. Capaz, C. Achete and A. Jorio, Carbon, 2010, 48, 1592-1597.

51 L. Liu, S. Ryu, M. R. Tomasik, E. Stolyarova, N. Jung, M. S. Hybertsen, M. L. Steigerwald, L. E. Brus and G. W. Flynn, Nano Lett., 2008, 8, 1965-1970.

52 S. Ryu, M. Y. Han, J. Maultzsch, T. F. Heinz, P. Kim, M. L. Steigerwald and L. E. Brus, Nano Lett., 2008, 8, 45974602.

53 S. Lee, J. R. Potts, J. Oh, J. Han, G. Park and S. Park, Solid State Sci., 2014, 27, 1-4.

54 F. Cataldo, O. Ursini and G. Angelini, Fullerenes, Nanotubes, Carbon Nanostruct., 2011, 19, 461-468.

55 A. Buchsteiner, A. Lerf and J. Pieper, J. Phys. Chem. B, 2006, 110, 22328-22338.

56 D. A. Dikin, S. Stankovich, E. J. Zimney, R. D. Piner, G. H. Dommett, G. Evmenenko, S. T. Nguyen and R. S. Ruoff, Nature, 2007, 448, 457-460.

57 B. E. Conway, Electrochemical Supercapacitors: Scientific Fundamentals and Technological Applications, Kluwer Academic/Plenum, New York, 1999.

58 S. W. Lee, N. Yabuuchi, B. M. Gallant, S. Chen, B. Kim, P. T. Hammond and Y. Shao-Horn, Nat. Nanotechnol., 2010, 5, 531-537.

59 S. K. Park, Q. Mahmood and H. S. Park, Nanoscale, 2013, 5, 12304-12309. 